\title{
Perspectives on immunotherapy utilization for hepatobiliary cancers in the United States
}

\author{
Pedro Luiz Serrano Usón Junior ${ }^{1,2}$, Daniel Ahn², Mohamad Bassam Sonbol², Tanios Bekaii-Saab ${ }^{2}$, \\ Mitesh J. Borad ${ }^{2}$ \\ ${ }^{1}$ Hospital Israelita Albert Einstein, São Paulo, Brazil; ${ }^{2}$ Mayo Clinic Cancer Center, Mayo Clinic, Phoenix, AZ, USA \\ Correspondence to: Mitesh J. Borad. Mayo Clinic Cancer Center, Mayo Clinic, Phoenix, AZ, USA. Email: Borad.mitesh@mayo.edu. \\ Provenance and Peer Review: This article was commissioned by the editorial office, Hepatobiliary Surgery and Nutrition. The article did not undergo \\ external peer review. \\ Comment on: Sahara K, Farooq SA, Tsilimigras DI, et al. Immunotherapy utilization for hepatobiliary cancer in the United States: disparities among \\ patients with different socioeconomic status. Hepatobiliary Surg Nutr 2020;9:13-24.
}

Submitted Nov 06, 2019. Accepted for publication Nov 30, 2019.

doi: 10.21037/hbsn.2019.11.20

View this article at: http://dx.doi.org/10.21037/hbsn.2019.11.20

Strategies involving immunotherapy and targeted therapies are emerging in the last years as valuable options for patients with hepatobiliary cancer (HBC) including hepatocellular carcinoma (HCC), gallbladder cancer (GBC), intrahepatic cholangiocarcinoma (ICC), and extrahepatic cholangiocarcinoma (ECC). For a long time, these diseases had only few treatment options and often with considerable toxicities $(1,2)$. Sahara and colleagues reported an analysis of 249,913 patients with HBC identified through the National Cancer Database (NCDB) from January 2004 to December 2015. Their main objective was to assess the utilization of immunotherapy (IO) in the treatment of HBC and examine socioeconomic and disease-related factors associated with the receipt of IO in the United States (US). As a result, the authors found that only a minority of patients received immunotherapy $(\mathrm{n}=585,0.2 \%)$. Some factors were statistically correlated with a higher chance to receive immunotherapy, including a more recent period of diagnosis (referent 2008-2011: 2012-2015), median income $\geq \$ 46,000$ (referent $<\$ 30,000$ ), diagnosis of HCC, ICC or ECC (referent: GBC), higher tumor stage, and prior receipt of cytotoxic chemotherapy. The authors have highlighted that socioeconomic factors were associated with an increased likelihood of receiving immunotherapy, which may suggest disparities in access or enrollment of patients with lower socioeconomic status (3).

Different IO therapies in biliary tract cancer (BTC) are currently under evaluation in prospective trials $(4,5)$. In KEYNOTE-028 (KN028) and KEYNOTE-158 (KN158) patients with incurable BTC who progressed on any number of prior standard treatment regimens have received pembrolizumab $200 \mathrm{mg}$ Q3W (KN158) or $10 \mathrm{mg} / \mathrm{kg}$ Q2W (KN028) for up to 2 years. No patient had MSI-H tumors and PD-L1-positivity (membranous PD-L1 expression in $\geq 1 \%$ of tumor and associated inflammatory cells or positive staining in stroma) was required for eligibility in $\mathrm{KN} 028$, but not in KN158. These studies were started in 2014 and 2015 respectively. In KN158, overall response rate (ORR) was $5.8 \%$, median overall survival (mOS) was 7.4 months, and median progression-free survival (mPFS) was 2.0 months. In KN028, the ORR was $13.0 \%$, the mOS and the mPFS were 6.2 months and 1.8 months, respectively (4). Nivolumab, another anti PD-1 monoclonal antibody, was evaluated in a phase II study started in 2016, in advanced refractory BTC. Patients who progressed on at least one line but no more than three lines of systemic therapy received nivolumab until disease progression or unacceptable toxicity. Of 45 patients, $22 \%$ achieved partial response and disease control rate was $60 \%$. After a median follow-up of 13.34 months, the mPFS was 3.98 months and the mOS was 14.22 months (5). Pembrolizumab was evaluated in mismatch repair deficient tumors in two cohorts, including BTC patients $(6,7)$. Objective radiographic responses were noted in $53 \%$ of all patients (46 of 86 patients; $95 \%$ CI: $42-64 \%)$, with $21 \%(n=18)$ achieving a complete radiographic response, objective response rate was similar between colorectal cancer versus other cancer subtypes (7). Currently, in the US, the approval for IO in BTC is only 
Table 1 FDA approvals for Immunotherapy in HCC and BTC in United States

\begin{tabular}{|c|c|c|c|c|c|c|}
\hline Immunotherapy FDA & \multicolumn{2}{|c|}{$\mathrm{HCC}$} & \multicolumn{2}{|c|}{ BTC } & \multicolumn{2}{|l|}{$\mathrm{HCC}$ and $\mathrm{BTC}$} \\
\hline Nivolumab & Yes & Sep 22, 2017 & No & $\mathrm{N} / \mathrm{A}$ & No & $\mathrm{N} / \mathrm{A}$ \\
\hline Pembrolizumab & Yes & Nov 9, 2018 & No & $\mathrm{N} / \mathrm{A}$ & Yes & May 23, 2017 \\
\hline
\end{tabular}

HCC, hepatocellular carcinoma; BTC, biliary tract cancer; N/A, not applicable.

for patients who have received standard frontline treatments (chemotherapy) in patients with microsatellite instabilityhigh (MSI-H) tumors.

In HCC, approval of nivolumab and pembrolizumab was based on prospective phase I/II studies, including patients who had received first-line treatment with sorafenib for advanced disease. CheckMate-040 is a prospective trial accessing nivolumab every two weeks in patients with advanced HCC, with or without chronic viral hepatitis. Previous exposure to sorafenib was allowed. Between November 26, 2012, and August 8, 2016, 262 patients were treated. The ORR ranged from $15 \%$ to $20 \%$, in doseescalation and dose-expansion (8). Comparable results were found in Keynote 224, evaluating pembrolizumab 200mg every 3 weeks (9). Between June 22, 2016, and February 20, 2017, 104 enrolled patients were treated with at least one dose of pembrolizumab and were included in the primary analysis. The ORR was $17 \%$ and disease control rate was $62 \%$. More recently, in the randomized phase III trial Keynote 240, pembrolizumab was compared with placebo (Pcb) in a group of patients previously exposed to sorafenib. After a median follow-up of 13.8 months, pembrolizumab improved OS (HR: 0.78; one sided $\mathrm{P}=0.0238$ ) and PFS (HR: 0.78; one sided $\mathrm{P}=0.0209$ ) versus $\mathrm{Pcb}$. However, these differences were not significant considering the prespecified statistical plan (10). More recently, at ESMO 2019, the results of the randomized phase III trial Checkmate-459 were reported (11). A total of 732 patients with advanced HCC were randomized to receive sorafenib or nivolumab in the first-line setting. With a minimum follow-up of 22.8 months, the mOS was 16.4 months for nivolumab and 14.7 months for sorafenib (HR 0.85; 95\% CI: 0.72-1.02; $\mathrm{P}=0.0752$ ). ORR was $15 \%$ for nivolumab and $7 \%$ for sorafenib. Grade 3/4 treatment-related adverse events were reported in $81(22 \%)$ patients in the nivolumab arm and 179 (49\%) patients in the sorafenib arm. Despite not reaching its prespecified primary endpoint for improved overall survival, the study showed a favorable safety profile with nivolumab compared to sorafenib.
Based on the results of the presented studies and the multivariate analysis of the data from the NCDB by the authors, it is clear that factors identified as being important which include a more recent diagnosis of disease, diagnosis of hepatocellular carcinoma, higher tumor stage, and the presence of previous systemic treatments are related to the characteristics of clinical trials developed and conducted during the analyzed period (2012-2015), which could have influenced off-label use of these drugs during this time (Table 1). For this reason, this analysis might be considered somewhat premature and a follow-up analysis will be important to assess the true rate of IO utilization in the current period. Higher income versus lower income differences could be related to off-label use (which would occur with greater frequency in privately insured patients). Interestingly, race is not a statistical positive factor and the distribution of demographics appears to be consistent with racial demographics of the United States population (12). Another issue to be addressed is the indications of adjuvant and neoadjuvant immunotherapy. In the study, 136 patients received IO and underwent surgery; and about 40-60\% did not have AJCC complete staging. There was a trend of IO use in 2004-2005 among patients who underwent surgery, where probably currently clinically relevant drugs (nivolumab and pembrolizumab), were not employed. Data on which drugs defined as IO were used during that period and the complete staging of these patients is needed in order to draw more precise conclusions regarding these indications. The trend in non-surgical patients with an increase in 2012 and peak by 2015 is possibly associated with clinical trials and subsequent drug approvals. Currently, there are no indications of immunotherapy in the adjuvant or neoadjuvant settings for HCC or BTC, outside of clinical trials. There are several ongoing trials addressing immunotherapy in the first-line and perioperative settings (Table 2). If any of these trials achieve positive results, there would likely be an uptrend in the use of immunotherapy given the differentiated safety profiles of immune checkpoint inhibitors to anti-angiogenic kinase 
Table 2 Clinic trials of checkpoint inhibitor therapy in hepatobiliary cancers

\begin{tabular}{|c|c|c|c|c|c|}
\hline Agents & Cancer type & Patient population & Immunotherapy target(s) & Phase & ClinicalTrials.gov locator \\
\hline Durvalumab and tremelimumab & $\mathrm{HCC}$ & Advanced & PD-L1 and CTLA-4 & Phase III & NCT03298451 \\
\hline Atezolizumab and bevacizumab & $\mathrm{HCC}$ & Advanced & PD-L1 & Phase III & NCT03434379 \\
\hline Pembrolizumab plus lenvatinib & $\mathrm{HCC}$ & Advanced & PD-1 & Phase III & NCT03713593 \\
\hline Nivolumab & $\mathrm{HCC}$ & Adjuvant & PD-1 & Phase III & NCT03383458 \\
\hline Pembrolizumab & $\mathrm{HCC}$ & Adjuvant & PD-1 & Phase III & NCT03867084 \\
\hline Durvalumab and bevacizumab & $\mathrm{HCC}$ & Adjuvant & PD-L1 & Phase III & NCT03847428 \\
\hline Durvalumab & BTC & Advanced & PD-L1 & Phase III & NCT03875235 \\
\hline KN035 & BTC & Advanced & PD-L1 & Phase III & NCT03478488 \\
\hline Nivolumab and TACE & $\mathrm{HCC}$ & Localized & PD-1 & Phase II & NCT03572582 \\
\hline Nivolumab and Y90 & $\mathrm{HCC}$ & Advanced & PD-1 & Phase II & NCT03033446 \\
\hline
\end{tabular}

HCC, hepatocellular carcinoma, BTC, biliary tract cancer, TACE, trans-arterial chemoembolization; Y90, Yttrium-90.

inhibitors. The authors developed an interesting analysis to elucidate the applicability issues of immunotherapy and HBC in the U.S. However, in the context of drug approvals in the recent years and likelihood of new indications in the upcoming years, a more up-to-date analysis of their use would be of interest to see if these socioeconomic disparities persist.

\section{Acknowledgments}

Funding: None.

\section{Footnote}

Conflicts of Interest: All authors have completed the ICMJE uniform disclosure form (available at http://dx.doi. org/10.21037/hbsn.2019.11.20). Dr. DA reports personal fees from Eisai, personal fees from Genentech, personal fees from Exelixis, outside the submitted work. Dr. TBS reports grants from Boston biomedical, grants and personal fees from Bayer, grants from Amgen, grants and personal fees from Merck, grants from Celgene, grants and other from Lilly, grants and personal fees from Ipsen, grants from Clovis, grants and personal fees from Seattle Genetics, grants and personal fees from Array biopharma, grants and personal fees from Genentech, grants from Abgenomics, grants and personal fees from Incyte, grants from BMS, personal fees from Boehringer Ingelheim, personal fees from TreosBio, personal fees from Sobi, other from Astra zenica, other from PanCan, other from 1Globe, other from Sun biopharma, other from Imugene, other from Immuneering, outside the submitted work; In addition, Dr. TBS has a patent WO/2018/183488 issued, and a patent WO/2019/055687 issued. Dr. MJB reports grants from Senhwa Pharmaceuticals, grants from Adaptimmune, grants from Agios Pharmaceuticals, grants from Halozyme Pharmaceuticals, grants from Celgene Pharmaceuticals, grants from EMD Merk Serono, grants from Toray, grants from Dicerna, grants from Taiho Pharmaceuticals, grants from Sun Biopharma, grants from Isis Pharmaceuticals, grants from Redhill Pharmaceuticals, grants from Boston Biomed, grants from Basilea, grants from Incyte Pharmaceuticals, grants from Mirna Pharmaceuticals, grants from Medimmune, grants from Bioline, grants from Sillajen, grants from ARIAD Pharmaceuticals, grants from PUMA Pharmaceuticals, grants from Novartis, grants from QUED Pharmaceuticals, grants from Pieris Pharmaceuticals, other from ADC Therapeutics, other from 
Exelixis Pharmaceuticals, other from Inspyr Therapeutics, other from G1 Therapeutics, other from Immunovative Therapies, other from OncBioMune Pharmaceuticals, other from Western Oncolytics, other from Lynx Group, other from Astra Zenica, outside the submitted work. The other authors have no conflicts of interest to declare.

Ethical Statement: The authors are accountable for all aspects of the work in ensuring that questions related to the accuracy or integrity of any part of the work are appropriately investigated and resolved.

Open Access Statement: This is an Open Access article distributed in accordance with the Creative Commons Attribution-NonCommercial-NoDerivs 4.0 International License (CC BY-NC-ND 4.0), which permits the noncommercial replication and distribution of the article with the strict proviso that no changes or edits are made and the original work is properly cited (including links to both the formal publication through the relevant DOI and the license). See: https://creativecommons.org/licenses/by-ncnd/4.0/.

\section{References}

1. Llovet JM, Ricci S, Mazzaferro V, et al. Sorafenib in advanced hepatocellular carcinoma. N Engl J Med 2008;359:378-390.

2. Valle J, Wasan H, Palmer DH, et al. Cisplatin plus gemcitabine versus gemcitabine for biliary tract cancer. New Engl J Med 2010;362:1273-81.

3. Sahara K, Farooq SA, Tsilimigras DI, et al. Immunotherapy utilization for hepatobiliary cancer in the United States: disparities among patients with different socioeconomic status. Hepatobiliary Surg Nutr 2020;9:13-24.

Cite this article as: Usón Junior PLS, Ahn D, Sonbol MB, Bekaii-Saab T, Borad MJ. Perspectives on immunotherapy utilization for hepatobiliary cancers in the United States. HepatoBiliary Surg Nutr 2020;9(4):501-504. doi: 10.21037/ hbsn.2019.11.20
4. Bang YJ, Ueno M, Malka D, et al. Pembrolizumab (pembro) for advanced biliary adenocarcinoma: Results from the KEYNOTE-028 (KN028) and KEYNOTE-158 (KN158) basket studies. J Clin Oncol 2019;37:4079.

5. Kim RD, Kim DW, Alese OB, et al. A phase II study of nivolumab in patients with advanced refractory biliary tract cancers (BTC). J Clin Oncol 2019;37:4097.

6. Le DT, Uram JN, Wang H, et al. PD-1 blockade in tumors with mismatch-repair deficiency. $\mathrm{N}$ Engl J Med 2015;372:2509-20.

7. Le DT, Durham JN, Smith KN, et al. Mismatch repair deficiency predicts response of solid tumors to PD-1 blockade. Science 2017;357:409-13.

8. El-Khoueiry AB, Sangro B, Yau T, et al. Nivolumab in patients with advanced hepatocellular carcinoma (CheckMate 040): an open-label, non-comparative, phase 1/2 dose escalation and expansion trial. Lancet 2017;389:2492-502.

9. Zhu AX, Finn RS, Edeline J, et al. Pembrolizumab in patients with advanced hepatocellular carcinoma previously treated with sorafenib (KEYNOTE-224): a non-randomised, open-label phase 2 trial. Lancet Oncol 2018;19:940-52.

10. Finn RS, Ryoo B, Merle P, et al. Results of KEYNOTE-240: phase 3 study of pembrolizumab (Pembro) vs best supportive care (BSC) for second line therapy in advanced hepatocellular carcinoma (HCC). J Clin Oncol 2019;37:4004.

11. Yau T, Park JW, Finn RS, et al. Checkmate 459: A randomized, multi-center phase 3 study of nivolumab (nivo) vs sorafenib (sor) as first-line (1L) treatment in patients (pts) with advanced hepatocellular carcinoma (AHCC). Ann Oncol 2019;30:mdz394.029.

12. Howlader N, Noone AM, Krapcho M, et al. SEER Cancer Statistics Review, 1975-2016, National Cancer Institute. Available online: https://seer.cancer.gov/csr/1975_2016/ 